Results: Mean disease duration was $11.8 \pm 10.5$ years for cancer and $12.8 \pm 10.8$ years for RMDs since diagnosis. $1.4 \%$ received cancer treatment $(6.4 \%$ of malignancy group), $69.3 \%$ csDMARDs, $42.3 \%$ b/tsDMARDs and $46.4 \%$ glucocorticoids at the time of sample collection.

Most metabolites tested were significantly lower in the malignancy groups versus associated controls: Concentrations of amino acids $V$ and $L$ were significantly reduced in all malignancy samples. Additionally, T, D, N, Q, E, A, I were altered in RA, SpA and PsA, changes in G were seen in RA, PsA and SLE and $P$ was altered in RA and PsA only. Furthermore, lower concentrations of short chain fatty acids and tricarboxylic acid cycle intermediates were present in the malignancy groups. In no case was a metabolite concentration significantly higher in the malignancy group than in the associated control. When comparing the metabolome within the four malignancy groups, only the concentrations of creatine, threonine and isoleucine were found higher in RA patients with malignancy.

Conclusion: Significant differences between the metabolomic fingerprints of RMD patients with and without malignancies could be observed. These changes might be characteristic for cancer burden, as in most cases the underlying RMD was not relevant when comparing the concentrations between the malignancy groups. Our results may promote understanding of the interrelationships of both disease entities as well as prove useful as biomarkers for diagnostic and therapeutic purposes.

Acknowledgements: Grant/research support from medical faculty (Olympia Morata Programme) and foundations commission (Herbert Daus estate) of University of Heidelberg

Disclosure of Interests: None declared

DOI: 10.1136/annrheumdis-2021-eular.2484

\section{POS0473 AUTOIMMUNITY AGAINST COLLAGEN IN RHEUMATOID ARTHRITIS (RA): SERUM LEVELS OF IGG AGAINST DENATURED TYPE II COLLAGEN SIGNIFICANTLY ELEVATED IN RA}

P. Drobinski ${ }^{1}$, E. Aighobahi ${ }^{2}$, A. C. Bay-Jensen ${ }^{3}$, M. Karsdal ${ }^{3} .{ }^{1}$ University of Copenhagen/Nordic Bioscience, Department of Biomedical Sciences, Copenhagen, Denmark; ${ }^{2}$ Technical University of Denmark, Department of Biotechnology and Biomedicine, Copenhagen, Denmark; ${ }^{3}$ Nordic Bioscience, ImmunoScience, Herlev, Denmark

Background: The presence of autoantibodies in Rheumatoid Arthritis (RA) is a hallmark of the disease and one of main criteria for diagnosis and clinical classification. Identification of anti-citrullinated protein antibodies (ACPA) and RF-Factors in circulation of RA patients remains the most characteristic and established criteria for diagnosis. However, due to diagnostic limitation of these biomarkers, only approximately $70 \%$ of patients with RA can be identified. As a consequence, there is a lack of diagnostic biomarkers for large number of patients. Diagnosis of these patients relies mainly on assessment of their clinical symptoms such as swollen and tender joints. This problem leaves a need for diagnostic improvement and development of more sensitive biomarkers.

Objectives: In this study, we aimed to develop a sensitive biomarker assay capable to identify and quantify presence of autoantibodies against denatured type II collagen in circulation of patients with RA.

Methods: The presence and levels of autoantibodies was measured in serum samples from 50 patients with moderate to severe RA who had inadequate response to Methotrexate (average age $51.2,86 \%$ of female, $78 \%$ of white race patients). Control cohort involved serum from 42 healthy age matched patients (average age $48.6,50 \%$ of female, $60 \%$ of white race patients). Denaturation of the type II collagen was performed by heat treatment for $15 \mathrm{~min}$ at $72{ }^{\circ} \mathrm{C}$. Improvement of assay sensitivity was investigated by measurements of autoimmunity levels against denatured and native type II collagen. Assay specificity was assessed by comparison of presence of autoantibodies against type II collagen versus albumin (non-sense control). The normality of data distribution was checked with Shapiro-Wilk test, significance between cohorts with Mann-Whitney nonparametric test and correlations with Spearman's correlation coefficient (rho).

Results: Serum levels of autoantibodies against denatured type II collagen were significantly higher in RA patients than in healthy controls $(P<0.0001)$ (Figure 1). We observed nearly 4 -fold difference between both cohorts. Denaturation of type II collagen showed high improvement of assay sensitivity and increased accessibility of collagen for binding of autoantibodies. Developed assay showed specificity for detection of type II collagen autoantibodies by displaying no levels of autoimmunity against other control proteins (albumin). The levels of serum autoantibodies correlated significantly $(P<0.03)$ with patient disease activity (DAS28) at baseline, displaying rho $=0.3$. Our assay showed good technical performance with acceptable inter- and intra- assay variations (18\% and $5 \%$ respectively).

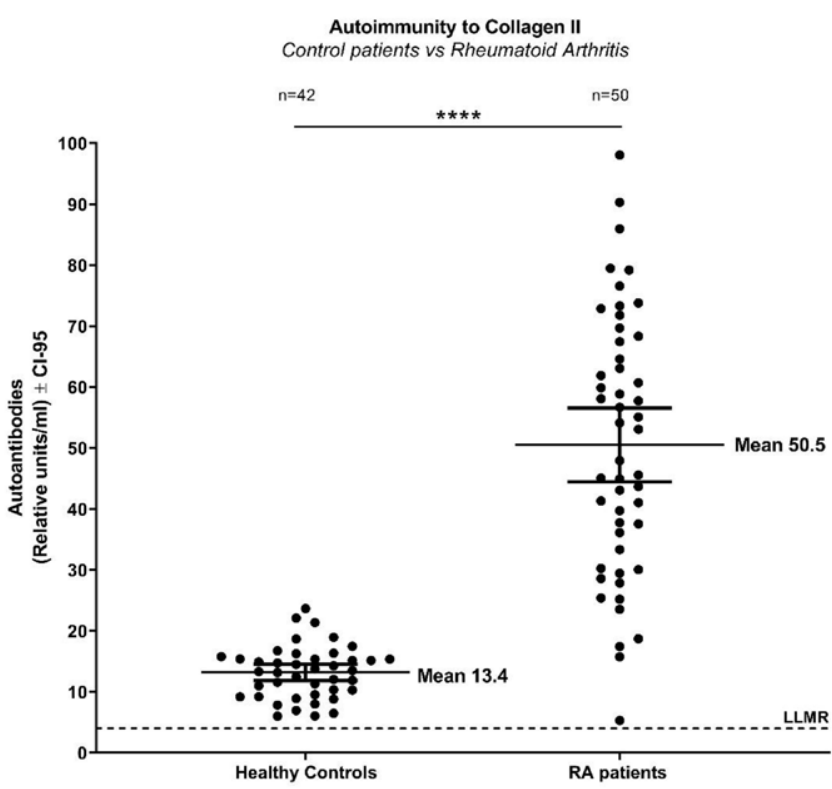

Figure 1. Differences in serum levels of autoantibodies against denatured type II collagen between healthy controls and patients with Rheumatoid Arthritis.

Conclusion: Present findings show that patients with RA carry upregulated levels of circulating autoantibodies directed against type II collagen. Heat-treatment of type II collagen increased exposure of immunogenic epitopes of collagen and enabled for more sensitive detection of autoantibodies directly in patient serum. Developed assay demonstrated potential for specific detection of autoantibodies and may provide additional diagnostic value in RA patients.

Disclosure of Interests: Patryk Drobinski: None declared, Elijah Aighobahi: None declared, Anne-Christine Bay-Jensen Shareholder of: Nordic Bioscience A/S, Employee of: Nordic Bioscience A/S, Morten Karsdal Shareholder of: Nordic Bioscience A/S, Employee of: Nordic Bioscience A/S DOI: 10.1136/annrheumdis-2021-eular.2488

\begin{tabular}{|l|l}
\hline POS0474 & SUBSTANTIAL IMPACT OF AUTOANTIBODY \\
& ENRICHMENT ON OUTCOMES IN EARLY \\
& RHEUMATOID ARTHRITIS TREATED WITH \\
& ABATACEPT: DATA FROM A LARGE POOLED \\
& ANALYSIS OF 4 RCTS
\end{tabular}

K. Michaud $^{1,2}$, S. S. H. Park ${ }^{3}$, K. Lozenski ${ }^{4}$, J. Vaile ${ }^{5}$, V. Khaychuk ${ }^{6}$, J. Pope ${ }^{7}$, P. G. Conaghan ${ }^{8,9} .{ }^{1}$ University of Nebraska Medical Center, Internal Medicine Division of Rheumatology, Omaha, NE, United States of America; ${ }^{2}$ FORWARD, The National Data Bank for Rheumatic Diseases, Wichita, KS, United States of America; ${ }^{3}$ Bristol-Myers Squibb, US HEOR, Princeton, NJ, United States of America; ${ }^{4}$ Bristol-Myers Squibb, Medical Data Generation, Princeton, NJ, United States of America; ${ }^{5}$ Bristol-Myers Squibb, Worldwide Medical, Princeton, NJ, United States of America; ${ }^{6}$ Bristol-Myers Squibb, US Medical Immunology and Fibrosis, Princeton, NJ, United States of America; ${ }^{7}$ Western University, Schulich School of Medicine, Division of Rheumatology, London, Ontario, Canada: ${ }^{8}$ University of Leeds, Institute of Rheumatic and Musculoskeletal Medicine, Leeds, United Kingdom; ${ }^{9}$ Leeds Teaching Hospitals NHS Trust, NIHR Leeds Biomedical Research Centre, Leeds, United Kingdom

Background: Biomarkers are commonly used as prognostic tools in rheumatoid arthritis (RA) and have additional potential to guide treatment decisions. Previous clinical trials of abatacept (ABA) such as the Early AMPLE trial (eAMPLE) and real-world data suggest differential treatment effects of $A B A$ based on the presence of biomarkers and disease stage..$^{1-4}$

Objectives: To supplement evidence of enhanced efficacy of ABA among patients with enriched autoantibody biomarkers and early disease stage by applying key inclusion criteria from the eAMPLE trial retrospectively to historic ABA RCTs.

Methods: Individual patient data (IPD) from four early-RA ABA trials (AGREE [NCT00122382], AMPLE [NCT00929864], AVERT [NCT01142726], AVERT-2 [NCT02504268]) were pooled. Patients were defined as enriched at baseline if they had: 1) RA disease duration $\leq 12$ months, 2) DAS28-CRP $\geq 3.2,3$ ) $\geq 3$ times the upper limit of normal on an anti-cyclic citrullinated peptide (anti-CCP) test, and 4$)$ were rheumatoid factor positive $(R F+)$. Patients were grouped according to treatment status (ABA [monotherapy or with methotrexate (MTX)] or non-ABA [MTX or adalimumab (ADA) plus MTX]). Outcomes included DAS28-CRP mean 